Canadian

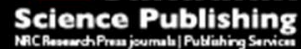

Canadian Journal of Microbiology Revue canadienne de de microbiologie

\title{
Mucosal and systemic immune responses induced by a Single Time Vaccination Strategy in mice
}

\begin{tabular}{|r|l|}
\hline Journal: & Canadian Journal of Microbiology \\
\hline Manuscript ID: & cjm-2015-0063.R1 \\
\hline Manuscript Type: & Article \\
\hline Date Submitted by the Author: & $29-$ Apr-2015 \\
\hline Complete List of Authors: & $\begin{array}{l}\text { Gonzalez, Elyzabeth; Finlay Institute } \\
\text { Romeu, Belkis; Finlay Institute and Permanent Mission of Cuba, Geneva, } \\
\text { Lastre, Miriam; Havana Medical School, } \\
\text { Zayas, Caridad; Finlay Institute } \\
\text { Cuello, Maribel; Finlay Institute } \\
\text { Cabrera, Osmir; Finlay Institute } \\
\text { Valdez, Yolanda; Finlay Institute } \\
\text { Fariña, Mildrey; Finlay Institute, } \\
\text { Pérez, Oliver; Havana Medical School, }\end{array}$ \\
\hline Keyword: & $\begin{array}{l}\text { vaccination strategy, adjuvants, nanoparticles, microparticles, mucosal } \\
\text { immunization }\end{array}$ \\
\hline
\end{tabular}


1 Mucosal and systemic immune responses induced by a Single Time Vaccination Strategy in

2 mice

3 Elizabeth González*, Belkis Romeu**1, Miriam Lastre ${ }^{2}$, Caridad Zayas, Maribel Cuello,

4 Osmir Cabrera, Yolanda Valdez ${ }^{3}$, Mildrey Fariñas ${ }^{3}$, and Oliver Pérez ${ }^{2}$

5 Immunology Department, Finlay Institute, P.O. Box 16017, Havana Cuba

$6 \quad{ }^{1}$ Permanent Mission of Cuba, Geneva

$7 \quad{ }^{2}$ Havana Medical School, Havana, Cuba

$8 \quad{ }^{3}$ Animal Models Direction, Finlay Institute. P.O. Box 16017, Havana Cuba

$9 *$ Authors with equal contribution

10 Corresponding authors:

11 Elizabeth González Aznar. Immunology Department, Finlay Institute. P.O. Box 16017, Havana

12 Cuba. elygonzalez@,finlay.edu.cu

13 Belkis Romeu, Finlay Institute and Permanent Mission of Cuba in Geneva. , Havana Cuba.

14 belkisorama@gmail.com 
24 Vaccination is considered by World Health Organization as the most cost-effective strategy for

25 controlling infectious diseases. In spite of great successes with vaccines, many infections

26 diseases are still leading killers, because of their inadequate coverage. Several factors have been

27 responsible: number of doses, high vaccine reactogenicity, vaccine costs, vaccination policy,

28 among others. Contradictorily, few vaccines are of single dose and even less of mucosal

29 administration. However, more common infections occur via mucosa, where secretory

30 immunoglobulin A plays an essential role. As an alternative, we proposed a novel protocol of

31 vaccination called Single Time Vaccination Strategy (SinTimVaS) by immunizing two priming

32 doses at same time, one by mucosal and one by parenteral routes. Here, the mucosal and

33 systemic responses induced by Finlay Adjuvants (AF Proteoliposome (PL) 1 and AF Cochleate

34 (Co) 1) implementing SinTimVaS in Balb/c mice were evaluated. One intranasal dose of AFCo1

35 and intramuscular dose of AFPL1 adsorbed onto Aluminum hydroxide, with BSA or TT as

36 model antigens, administrated at the same time, induced potent specific mucosal and systemic

37 immune responses. Also, we demonstrated that SinTimVaS using other mucosal routes like oral

38 and sublingual, in combination with subcutaneous route elicits immune responses. SinTimVaS,

39 as a new immunization strategy, could increase vaccination coverage and reduce time-cost

40 vaccines campaigns, adding the benefits of immune response in mucosa.

42 Keywords: adjuvants, mucosal immunization, vaccination strategy, cochleates, outer membrane 43 vesicles 


\section{Introduction}

48 Vaccination is considered by the World Health Organization (WHO) as the most cost-effective 49 strategy for controlling infectious diseases that prevent 2.5 million of deaths every year (WHO, 50 2005). Despite of the great success of vaccination, many infectious diseases are still leading 51 killers, mainly due to their insufficient vaccination coverage (WHO, 2009; Drain et al. 2003). A 52 number of factors have been responsible of the logistical challenges to attain high immunization 53 coverage: required doses on immunization schedules, parenteral immunization, high vaccine 54 reactogenicity, few studies targeting antigens-combinations on vaccine formulations, vaccine 55 cold chain, vaccination policy, countries weak immunization programmes, high cost and not 56 affordability for poor countries, among others (Jiang et al. 2005). Only live-attenuated vaccines 57 like BCG and Polio induce good protection with a single dose. In contrast, to obtain good immune response with a single dose by non-living antigen vaccines multiple doses are generally

59 needed, in order to provide sufficient stimulation of the immune system and to achieve long-

60 lasting immune responses. The number of doses is especially relevant in developing countries,

61 where the population has not easy access to medical services and vaccination campaigns are 62 very difficult, much more when several doses are required, which cause loses the entire immunization schedule (Aguado and Lambert 1992). So, the development of new immunization

64 strategies that allow complete immunization scheme with a single visit without further contact 65 could be as important as to develop single-dose vaccines.

66 Traditionally, adjuvants have been used to enhance the magnitude of the adaptive response to a 67 vaccine. However, a second role has become increasingly important: guiding the type of adaptive 68 response to produce the most effective forms of immunity for each specific pathogen (Th1, Th2, 69 Th17, Th9) (Coffman et al. 2010). Adjuvants have been classified as: immunopotentiators, 70 delivery systems (O'Hagan and Singh 2003), and immunopolarizators(Perez et al. 2013) and 71 neither of these activities are mutually excluding, even, some adjuvants exhibit at least two of 
72 them. The use of potent adjuvants that exhibit several mechanisms of action and enabling 73 comparable immune responses using substantially lower antigen amount and doses could be very 74 important to develop novel vaccination strategies.AFPL1 (Adjuvant Finlay Proteoliposome 1), a nanoparticle derived from the outer membrane of Neisseria meningitidis serogroup B, has been widely used as the main antigen of the Cuban meningococcal vaccine administrated in more that 60 million doses in humans (Campa et al. 1997 and Perez et al. 2001). AFCo1 (Adjuvant Finlay Cochleate 1), is a microparticle with cochlear structure obtained from the interaction of anionic lipid from AFPL1 with divalent cations, like $\mathrm{Ca}^{2+}$ (Perez et al. 2004)). Both structures combine in the same structure: 1) immunepotentiator activity, containing major outer membrane proteins including Por B, lipopolysaccharide (LPS), phospholipids and others synergistic microorganismassociated molecular patterns (MAMPs) like traces of DNA; 2) immunopolarizer actions, inducing a preferential Th1 pattern; and 3) delivery system capacity, based on lipids presence (Del Campo et al. 2009). Their potential as adjuvants has been tested with a variety of antigens: Plasmodium falciparum merozoite surface protein 4 and 5 (Bracho et al. 2009), Herpes Simplex Virus type 2 protein (Del Campo et al. 2010), Streptococcus pyogenes proteins (Guillherme et al. 2009) and purified Dermatophagoides siboney allergens (Lastre et al. 2006). Both structures also induce cross-presentation, and cytotoxic T-lymphocyte activity (CTL) (Rodriguez et al. 2005 and Rodriguez et al. 2006). AFCo1 can be used mainly as mucosal adjuvant because it is a stable particle with higher mucosal absorption (Del Campo et al. 2009) and acid resistant properties.

Mucosal surface is exposed to a diverse and very large number of microorganisms and also is the

92 site of infection of diverse pathogens (e.g., influenza virus, Vibrio cholerae, type I reovirus, and 93 rhinovirus) (Plotkin, 2005). The initial mucosal infection can only be prevented by mucosal 94 immune responses (pathogen-specific secretory IgA (SIgA) or local cytotoxic T lymphocytes 95 (CTL)) (Brandtzaeg, 2009). Mucosal immunization elicits systemic specific immune response and stimulates the specific mucosal immune response, leading by the production of SIgA 
97 antibody. Also, it is less invasive route; does not require trained personnel; and significantly

98 decreases the risk of parenteral transmitted diseases (Mann et al. 2009; Neutra and Kozlowski, 99 2006). In our experience mucosal route requires larger antigen amount and number of doses than 100 parenteral route to obtain similar systemic responses (Del Campo et al. 2009). In addition, 101 parenteral immunization has been shown induction of memory IgA in different vaccines, such as 102 influenza, Pseudomonas aeruginosa, and inactivated polio vaccines (attributed to previous 103 natural mucosal infection by these organisms) (Kaul and Ogra, 1998). Indeed, a combined 104 mucosal and parenteral poliovirus immunization strategy has been shown to effectively induce 105 high neutralizing antibodies in serum as well as mucosal immune responses in humans. 106 Strategies which combine together parenteral and mucosal immunizations (Treanor et al. 1992; 107 Morrison et al. 1998), such as mucosal prime-parenteral booster (Lee et al. 1999; Faden et al. 108 1990) or parenteral prime-mucosal booster (29Keren et al. 1988) have generated stronger 109 immune responses than either of these routes by themselves.

110 In the light of the ambitious goal of vaccines providing maximum protection at both parenteral 111 and mucosal levels with minimum number of doses; we set out to develop a novel vaccination 112 strategy, called Single Time Vaccination Strategy (SinTimVaS) (Perez et al. 2008; Gonzalez et 113 al. 2009). SinTimVaS combines two priming (one mucosal and one parenteral) at the same time 114 without subsequent boost; being the presence of potent adjuvants one of the primary goals. In 115 this work, we assessed the ability of SinTimVaS to induce an efficient systemic and mucosal 116 immune response in mice against bovine serum albumin (BSA) protein and Tetanus Toxoid (TT)

117 by different mucosal and parenteral immunizations routes. We showed that mice immunized 118 with one AFCo1 intranasal (i.n) dose and one AFPL1 intramuscular (i.m) dose at the same time 119 combined with BSA or TT antigens triggered specific serum IgG and mucosal IgA immune 120 response against these specific antigens. Furthermore, we demonstrated that the new strategy 121 also works for oral (o.r), sublingual (s.l), and subcutaneous (s.c) routes combinations. 
123 Materials and methods

\section{Animals}

125 The experiments were assessed using female Balb/c mice from National Center for the

126 Production of Laboratory Animals (CENPALAB), Havana, Cuba of 8-10 weeks of age and 16-

$12718 \mathrm{~g}$ of weight. Mice were randomly distributed into five mice per experimental groups or

128 control groups. All mice were kept in ventilated cages, water ad libitum under specific conditions

129 at the Finlay Institute Animal Research Facilities. All animal studies were carried out following

130 the guidelines for the use of Laboratory Animals (EEC Council Directive, 1987) and were

131 approved by the Ethics Committee for Laboratory Animals in Havana, Cuba.

\section{Adjuvants and antigens}

133 TT and AFPL1, of the epidemic meningococcal strain Cu385 (B:4:P1.19,15), were produced at 134 industrial scale under Good Manufacturing Practices (GMP) conditions by manufacturing plants 135 at Finlay Institute, Havana, Cuba (9). AFPL1 was absorbed onto aluminium hydroxide (Alum).

136 AFCo1 was obtained in our laboratory starting from PL, through a process of exchange of 137 sodium deoxycholate (DOC) and the divalent cation calcium as described by Pérez et al (8). The 138 formation of cochleate structure was visualized by the appearance of a white suspension in the 139 preparation and by light microscopy analysis. BSA protein was supplied from Sigma, Aldrich.

\section{$140 \quad$ Immunization schedules}

141 Groups of 5 female mice were immunized simultaneously by i.m injection of AFPL1 (12.5 $\mu \mathrm{g})$ 142 or by mucosal (i.n, s.l, or o.r) administration of AFCo1 $(50 \mu \mathrm{g})$. Also, groups of mice were 143 immunized 2 times (days 0 and 14) i.m with AFPL1 (12.5 $\mu \mathrm{g}$ ), and 3 times (days 0,7 and 14) 144 i.n $(12.5 \mu \mathrm{L} /$ nostril $)$ with $\mathrm{AFCo} 1(50 \mu \mathrm{g})$. With heterologous antigens, mice were immunized 145 using the same scheme described above. Each dose contained BSA (10 $\mu \mathrm{g}$ per mouse per dose) 146 plus AFPL1 (12.5 $\mu \mathrm{g})$ or TT (2Lf (limit to flocculation) per dose per mouse) plus AFPL1 (12.5 
$147 \mu \mathrm{g})$ on i.m or s.c immunizations. In i.n, s.l and o.r immunizations, each dose contained BSA (20

$148 \mu \mathrm{g}$ per dose per mouse) or TT (5 Lf per dose per mouse) plus AFCo1 (50 $\mu \mathrm{g})$. The volume

149 delivered in each dose was: $12.5 \mu \mathrm{L}$ per nostril for i.n, $10 \mu \mathrm{L}$ under the tongue for s.1, and 100

$150 \mu \mathrm{L}$ for o.r routes. Groups of animals receiving saline were used as experimental control for

151 immunization. Table 1 shows the different SinTimVaS combinations used.

\section{Samples collection}

154 Samples were collected as follows. saliva and faeces were collected on the 7 day after the last 155 dose, serum and vaginal washes on the 21 st days after the last dose (12-30). Saliva samples were 156 taken following salivation stimulation with $50 \mu \mathrm{L}$ intraperitoneal injection of pilocarpine $(0.5 \%$ 157 (v/v) Quimefa, Cuba). Saliva was inactivated for $15 \mathrm{~min}$ at $56^{\circ} \mathrm{C}$, centrifuged at $10000 \mathrm{~g}$ for 10 158 min and stored until it was used. Three to 6 pieces freshly voided faeces were collected into 1.5 $159 \mathrm{~mL}$ pre-weighed micro-centrifuge tubes and phosphate buffered saline (PBS, pH 7.2) was added 160 with protease inhibitors ( $1 \mathrm{mM}$ phenylmethyl sulfonylfluoride in ethanol, $5 \mu \mathrm{g} / \mathrm{mL}$ of aprotinin 161 and $1 \mu \mathrm{L} / \mathrm{mL}$ of leupeptin, antipain and pepstatin (all from Sigma) in a ratio of $20 \mu \mathrm{L}$ per mg of 162 faeces. Solid matter was resuspended by extensive vortex and centrifuged at $10000 \mathrm{~g}$ for $10 \mathrm{~min}$ 163 and the supernatants were stored. Vaginal cavities were rinsed 3 times with $50 \mu \mathrm{L}$ of PBS, 164 centrifuged and immediately stored. Blood was collected by puncturing the retro-orbital plexus 165 using microhematocrit capillary tubes. The serum was removed by centrifugation at $5000 \mathrm{~g}$ for 10 $166 \min$. All samples were stored at $-20^{\circ} \mathrm{C}$ until ELISA's assays were conducted.

168 Analysis of immune response by Enzyme-linked immunosorbent assay (ELISA)

169 Anti PL, BSA, or TT-specific IgA, IgG, IgG1, and IgG2a antibodies were measured. Briefly, 96170 well ELISA plates (Nunc MaxiSorp F96; Roskilde, Denmark) were coated overnight at $4^{\circ} \mathrm{C}$ with $171100 \mu \mathrm{L} /$ well with: PL $(20 \mu \mathrm{g} / \mathrm{mL})$, BSA $(10 \mu \mathrm{g} / \mathrm{mL})$ or TT $(5 \mathrm{Lf} / \mathrm{mL})$ in carbonate bicarbonate 
172 buffer (pH 9.6). After blocking with PBS-1\% Gelatin (Blocking Solution (BS)) and incubated at

173 room temperature for 1 hour, plates were washed with PBS containing $0.05 \%$ Tween-20. Then,

174 samples were diluted in BS as following: 1:2 for saliva and feces, 1:10 for vaginal washes and

$1751: 100$ for sera, adding $100 \mu \mathrm{L} /$ well and incubated $2 \mathrm{~h}$ at $37^{\circ} \mathrm{C}$. The plates were incubated at $37^{\circ} \mathrm{C}$

176 for $2 \mathrm{~h}$ with the different conjugates: anti-mouse IgA biotinylated antibody $(4 \mu \mathrm{g} / \mathrm{mL}$, Sigma,

177 USA), anti-mouse IgG peroxidase-conjugated antibodies (1:5000, Sigma, USA), and anti-mouse

178 IgG1 and IgG2a biotinylated antibody (1:2500, Sigma, USA) for IgG isotypes. Streptavidin

179 peroxidase-conjugate (1:2000 dilution, Sigma, USA) was added and incubated for $2 \mathrm{~h}$ at $37^{\circ} \mathrm{C}$.

180 Each plate was developed with O-phenylene diamine (OPD) (Sigma, USA) in citrate-phosphate

181 buffer $\mathrm{pH} 5.0$ and the reaction was stopped with $\mathrm{H} 2 \mathrm{SO} 42 \mathrm{M}$. Optical densities (OD) were read

182 at $492 \mathrm{~nm}$ and the results were expressed as OD. Anti PL IgG and IgA titters were expressed as

183 arbitrary units per $\mathrm{mL}(\mathrm{AU} / \mathrm{mL})$ referred to an in-house standard. The cut-off value was

184 established as the mean OD 492 plus two times the standard deviation (SD) of control group. In

185 all plates a positive and negative sera were included to validate the assay.

186

187 Statistical analysis

188 Data were analyzed using the GraphPAD prism software (San Diego, CA, USA). The statistical

189 significance of the difference between group means was analyses by Student's two-tailed $t$-test

190 for two groups, or by one-factor analysis of variance (ANOVA) followed by Tukey's test for

191 three or more groups. Differences were considered significant with $P<0.05$.

192

193 Results

194 SinTimVaS applied by intranasal / intramuscular routes induces anti PL mucosal and 195 systemic immune responses 
196 To determinate if SinTimVaS (i.n/i.m) might induce systemic and mucosal immune response,

197 this novel strategy with the traditional immunization schedule of 3 i.n and 2 i.m doses were

198 compared. Groups of Balb/c mice were primed mucosally (i.n) and systemically (i.m) at the

199 same time without subsequent booster. As AFCo1 and AFPL1 have conserved in their structure

200 several protective outer membrane proteins, we can determinate immune responses against their

201 self. Twenty one days after the last dose, the levels of systemic and mucosal immunity were

202 evaluated by determining serum anti PL IgG and IgA responses. The serum anti PL IgG induced

203 by SinTimVaS were similar to those induced by 3 i.n doses of AFCo1 or 2 i.m doses of AFPL1

204 (Fig. 1A), maintaining the same subclasses behaviour (Fig. 1B).. Thus, the observed levels of

205 IgG2a in mice immunized by different SinTimVaS combinations suggest a Th1 polarization

206 (Fig. 2B) as is the case of the immune response of mice immunized with 3 i.n or 2 i.m doses. In

207 saliva, anti-PL IgA was significant higher $(\mathrm{p}<0.001)$ than 2 i.n or 2 i.m doses of AFCo1 and

208 AFPL1, respectively. In addition, neither one i.n nor 2 i.m doses induce specific IgA (Fig. 1C).

209 Overall, SinTimVaS overcome the several required doses for mucosal immune response

210 induction when it is supplemented with a simultaneous parenteral dose.

\section{SinTimVaS works with other mucosal routes}

212 In order to evaluate if SinTimVaS can be extended to other mucosa routes, s.1/i.m and o.r/i.m 213 combination were administrated and compared to traditional immunization schedules.

214 SinTimVaS combination of s.l/i.m or o.r/i.m priming induce systemic immune response 215 significant higher $(\mathrm{p}<0.01)$ than 3 s.1 and 3 o.r doses of AFCo1 (Fig. 2A). We also tested specific 216 IgG subclasses in the sera of immunized mice, and found that IgG1 and IgG2a were induced in 217 all SinTimVaS combinations (Fig. 2B). In addition, the presence of high levels of IgG2a in sera 218 constitutes direct evidence of Th1. The anti PL IgA response was detected in all mucosal 219 samples (saliva, feaces and vagina). Both SinTimVaS combinations (s.1/i.m and o.r/i.m) induce 220 similar mucosal specific IgA in all mucosal samples when we compared with 3 s.l and 3 o.r 
221 doses of AFCo1. Nevertheless, anti-PL specific IgA response induced by i.n route is higher to 222 other mucosal routes (Fig. 2C). Lastly, mucosal specific IgA was induced not only at the regional 223 site of immunization but also at distal places like the vagina and intestine when i.n and s.l routes 224 were used in SinTimVaS combinations. These results confirmed that SinTimVaS works even 225 when other mucosal routes of immunization were used.

226 Mucosal and systemic immune response induced by SinTimVaS with subcutaneous route 227 as parenteral priming.

228 To determinate whether the s.c route as parenteral priming in SinTimVaS induces systemic and 229 mucosal immune responses, we compared different combinations of mucosal (i.n, s.l, o.r) routes 230 and s.c route with 3 mucosal doses of AFCo1 and 2 s.c doses of AFPL1(Fig. 3). At systemic or 231 mucosal levels not differences were observed when s.c priming was used. Also, all SinTimVaS 232 combinations were capable to induce high levels of IgG1 and IgG2a. These results demonstrated 233 that this novel strategy of immunization also works with s.c route as parenteral priming.

234 Mucosal and systemic immune responses induced by SinTimVaS when AFPL1 and AFCo1 235 were used as adjuvants against BSA and TT.

236 To address if Finlay Adjuvants (AFPL1, to parenteral routes and AFCo1 to mucosal routes) are 237 able to elicit immune response against two model antigens (BSA and TT) using the proposed 238 strategy, mucosal and systemic anti-BSA and anti-TT immune responses were examined.. 239 Figure.4A and B show anti-BSA specific IgG in sera and IgA in saliva, respectively. 240 SinTimVaS i.n/i.m combination induced superior response anti-BSA $\operatorname{IgG}(\mathrm{p}<0.05)$ than s.1/im or 241 o.r/i.m combinations (Fig. 4A). Also, the response at the systemic level in all SinTimVaS 242 combinations were significantly superior $(p<0.05)$ to 3 doses of each mucosal route. Figure 4B 243 show that we detected a slight increase in anti-BSA IgA response in mice immunized with 244 i.n/i.m and o.r/i.m combinations when compared with each only-mucosal scheme, respectively. 245 Figure 4C shows anti-TT systemic response induced by SinTimVaS combinations.. Furthermore, 
246 the anti-TTspecific IgG in sera of o.r/i.m and s.1/i.m combinations was stimulated in comparison

247 with antibodies levels induced by 3 oral or sublingual doses. Moreover, anti-TT IgG response

248 induced by i.n/i.m combination was similar to those induced by 3i.n doses of AFCo1-TT or 2 i.m

249 doses of AFPL1-TT. However, in saliva anti-TT response of SinTimVaS combinations was not

250 superior to the response induced by 3 doses of AFCo1-TT by different routes (Fig. 4D), but it

251 was significantly higher $(\mathrm{p}<0.05)$ than the IgA detected in mice immunized with 3 doses of TT

252 alone (data not show). These results indicate that Finlay adjuvants in SinTimVaS are highly

253 effective in eliciting the production of both mucosal and systemic immune responses against

254 heterologous antigens.

\section{Discussion}

256 SinTimVaS constitutes a novel alternative to solve the problems of current catch-up vaccines

257 campaigns, which are so difficult to implement in large countries with weakened health systems.

258 Simultaneous immunization of one mucosal dose (i.n, s.l, o.r) and one parenteral dose (i.m, s.c)

259 as the complete immunization scheme induces efficient systemic and mucosal responses, similar

260 to those obtained by two only-parenteral or three only-mucosal immunizations, respectively. .

261 The Global Immunization Vision and Strategy 2006-2015, elaborated by WHO Department of

262 Immunization, proposed to carry out in a short-term period, the introduction of new vaccines,

263 technologies and strategies, focusing on the need to increase vaccination coverage and reduce the

264 morbidity and mortality from infectious diseases. More research on less expensive immunization

265 strategies should be prioritized on the view to reducing cost-effectiveness of vaccines.

266 Sustainable systems for children, adolescents, and adults vaccination must be developed (CDC,

267 2005). In this sense, the importance of SinTimVas lies on the possibility for providing better

268 vaccination coverage: achieving that in a single visit, the full immunization schedule is covered

269 and booster doses are not needed. Our vaccination regime induced high titers of IgG2a, even

270 comparable to the levels obtained when mice were vaccinated with the 3 nasal doses of AFCo1 
271 or 2 i.m doses of AFPL1. An induction of IgG2a antibodies generally indicates Th1 immunity,

272 whereas the induction of IgG1 antibodies is easier to induce and is not a conclusive indication of

273 a Th2 response.

274 Initial work of a consecutive immunization strategy, known as "prime-boost" were developed 275 with DNA vaccines; involving priming with DNA and boosting with vaccines encoding multiple 276 common HIV antigens. Prime-boost vaccination strategies amplify T-cell immunity and 277 ameliorate AIDS in macaques (Dale et al. 2006). In our study, the combination of only-one 278 parenteral dose (i.m or s.c) reinforced by a simultaneous subsequent mucosal dose, induce 279 efficient mucosal immune response. The levels of with secretory IgA were as high as the 280 sequential mucosal doses (one week apart from each other). Additionally, the use of other 281 mucosal routes (s.1 or o.r) in the strategy elicited strong systemic and mucosal responses, with 282 SIgA detected in saliva, faeces or vaginal washes. Furthermore, it is known that AFPL1 can 283 activate the maturation of dendritic cells (DC) and macrophages (Rodriguez et al. 2005). Thus, 284 the maturation of DC might have contributed to the strong mucosal responses detected. Vajdy y 285 cols., 2003 found that oral or intranasal followed by i.m. immunizations induced significantly 286 higher serum titres against H. pylori antigens (NAP and CagA) compared to i.n. alone, oral alone 287 or i.m. alone (Vajdy et al. 2003). More importantly, they found that while both i.n. and i.m. recall 288 immunizations induced similar levels of serum antibody responses, mucosal IgA responses in 289 faeces were only achieved through i.n. recall immunization (Vajdy et al. 2003).

290 This novel vaccination approach also showed that mice immunized by subcutaneous route can 291 induce potent antibody responses at mucosal and systemic compartments. However, in the s.c 292 route the antigen is administrated to the adipose tissue while in the i.m route the antigen delivery 293 is directly to the muscle, which allows a sustained absorption of the antigen. Patrolling DCs are 294 also numerous in the well-vascularized muscles and they are fewer in adipose tissues (Balasse et 295 al. 2008), therefore it could be hypothesized that i.m injection in SinTimVaS may be considered 
296 as a priming dose. . Moreover, both adjuvants have potent activity as immunepotentiators, given

297 by the MAMPs presented in their structures. The recognition of these MAMPs by TLRs, leads to

298 activation of a complex system of signal transduction, leading to the maturation and activation of

299 DC. Previous studies by our group have demonstrated the potent DC activation by the AFPL1,

300 evidenced by a high production of pro-inflammatory cytokines (IL-1ß, IL-6, TNF and IL-12)

301 (Perez et al. 2001) and by the high expression in DC of co-stimulatory molecules CD40, CD80

302 and CD86 (Rodriguez et al. 2005). Immunized MyD88 deficient mice with AFCo1 and

303 glycoprotein D of HSV-2 by intranasal route were unable to produce specific proliferative and

304 IFN- responses of splenic CD4+ cells. The presence of TLR adaptor molecule MyD88 has

305 demonstrated to be necessary to induce a potent IgG response and protect against challenge with

306 herpes simplex virus type 2 (HSV-2) (Del Campo et al. 2010). AFCo1 and AFPL1, contains

307 major outer membrane proteins of $N$. meningitidis B, including LPS and porins (PorA and PorB)

308 (Del Campo et al. 2009) that can activate TLR4 and TLR2, respectively. While TLR2 transduces

309 signal exclusively through the adaptor molecule MyD88, LPS exerts its immunopotentiating

310 effect through a MyD88-dependent as well as a MyD88 independent TIR-domain-containing

311 adapter-inducing interferon- (TRIF)-based pathways (Perez et al. 2012).

312 More importantly, we have shown that SinTimVas is capable of activating immune response

313 against heterologous antigens when Finlay Adjuvants are incorporated in the combination. The

314 SinTimVaS approach induced high specific anti-heterologous antibodies titers in sera and

315 mucosal compartments similar to two-parenteral weekly spaced doses or several weekly spaced

316 mucosal doses. It is well known that parenteral immunization usually elicits a peripheral immune

317 response, with protective IgM/IgG antibodies and peripheral cell-mediated immunity but fails to

318 stimulate mucosal lymphatic tissues to generate protective IgA antibodies (Lawson et al. 2011).

319 Meanwhile, mucosal immunization confers immunity at the directly vaccine-exposed mucosa

320 followed by the adjacent mucosal tissues. Also, mucosal tissues are interconnected via 
321 circulating lymphocytes; immunization at one mucosal inductive site may induce an immune

322 response at other, even remote, mucosal surface (Puga et al. 2010). The mucosal efficacy of

323 AFCo1 appears to owing a directly engagement with B cells or other antigen presenting cells.

324 Direct TLR stimulation enhances T independent IgA class switching. As the antigens immunized

325 by parenteral routes reach the lymphatic pathway earlier than those who are immunized by

326 mucosal routes, we can hypothesize that in SinTimVaS, mucosal immunization can works as a

327 booster of the parenteral immunization.

328 Small-sized particles as vaccine delivery systems are efficient in permeating biological barriers

329 and affecting the kinetics of lymphoid drainage (Singh et al. 2007). In our strategy, a

330 combination of nanoparticle and microparticle was used simultaneously. AFPL1, a nanometric

331 particle of $70 \mathrm{~nm}$ was applied by parenteral immunization. Nanoparticles of less than $200 \mathrm{~nm}$

332 reach the lymphoid organs directly through the lymph drainage within hours of injection,

333 whereas the transport of particles larger than 200-500 $\mathrm{nm}$ requires DCs, and it takes

334 approximately 24 hours for them to arrive in lymph nodes (Jennings et al. 2007; Bachmann and

335 Jennings, 2010). On the other hand, AFCo1, a microparticle with $\approx 11 \mu \mathrm{m}$ of length, was applied

336 by mucosal route. There are reports showing that particles in the size range of $2-8 \mu \mathrm{m}$ were

337 capable to induce after a single-point i.m immunization high antibody titers, whereas particles of

$33850-150-\mu \mathrm{m}$ of length generated weaker antibody titers (Bachmann and Jennings, 2010). Also,

339 data from other studies reported considerable antibody responses even from large microparticles

340 of $10-90-$ and $15-60-\mu \mathrm{m}$ size ranges (41). Finally, results from our group have shown that

341 SinTimVaS using ovalbumin as model antigen is capable to induce systemic anti-Ova $\operatorname{IgG}$

342 response and long-lasting memory response, when a booster of ovalbumin at day 125 was

343 administrated (Perez et al., 2013).

344 In summary, two priming applied at the same time by different immunization routes can induce

345 efficient mucosal and systemic responses. Undoubtedly, the strength of the new vaccination 
346 strategy (SinTimVaS) is to improve vaccination coverage, reducing the logistics and the number

347 of follow-up and catch-up campaigns. SinTimVaS could solve non-continuity vaccination

348 problems, allowing a complete - vaccination scheme in one immunization service. Further

349 studies to explain the mechanisms involved in the activation of the immune response by this

350 strategy are needed. Greater understanding of SinTimVaS mechanism to increase and sustain

351 immunization levels is necessary in order to create long-lasting responses. Other issues to

352 consider are the application of SinTimVaS to other parenteral and mucosal adjuvants and to

353 evaluate long-lasting immune responses against other antigens.

354 Declaration of interest. The authors report no conflicts of interest. The authors are sole 355 responsible for the content and writing of the paper.

356 Acknowledgments. We would like to thank Finlay Institute for the support and sponsorship of 357 this work. We thank Dr. Luis Garcia, Research Deputy Director of Finlay Institute and Dr. 358 Reinaldo Acevedo, Basic Research Group Leader for supporting this work. We would like to 359 thank Patricia Borrego for its assistance in English.

\section{References}

Aguado, M.T. and Lambert, P.H. 1992. Controlled-release vaccines-biodegradable polylactide polyglycolide $(\mathrm{Pl} / \mathrm{Pg})$ microspheres as antigen vehicles. Immunobiology. 184(2-3):113-25.

Bachmann, M.F. and Jennings, G.T. 2010. Vaccine delivery: a matter of size, geometry, kinetics and molecular patterns. Nat Rev Immunol. 10(11):787-96. doi: 10.1038/nri2868. 
Balasse, E., Odot, J., Gatouillat, G., et al. 2008. Enhanced immune response induced by BSA loaded in hydroxyethylstarch microparticles. Int J Pharm. 353(1-2):131-8. Brandtzaeg, P. 2009. Mucosal Immunity: Induction, Dissemination, and Effector Functions. Scand J Immunol. 70(6):505-15. doi: 10.1111/j.1365-3083.2009.02319.x. Campa, C., Sierra, V.G., Gutiérrez, M.M., et al. 1997. Method of producing Neisseria meningitidis B vaccine, and vaccine produced by method. United States Patent, Patent number $5597572 \mathrm{~A}$.

CDC. 2005. Immunization Strategies for Healthcare Practices and Providers. This publication is available on the Internet at: www.cdc.gov/vaccines/pubs/pinkbook/ Coffman, R.L., Sher, A., Seder, R. A. 2010. Vaccine Adjuvants: Putting Innate Immunity to Work. Immunity.33(4):492-503. doi: 10.1016/j.immuni.2010.10.002.

Dale, C.J., Thomson, S., De Rose, R., et al. 2006. Prime-boost strategies in DNA vaccines. J Methods Mol Med. 127:171-97.

Del Campo, J., Lindqvist, M., Cuello, M., et al. 2010. Intranasal immunization with a proteoliposome-derived cochleate containing recombinant $\mathrm{gD}$ protein confers protective immunity against genital herpes in mice. Vaccine. 28(5):1193-200. doi: 10.1016/j.vaccine.2009.11.035.

Del Campo, JD, Zayas, C, Romeu, B, et al. 2009. Mucosal immunization using proteoliposome and cochleate structures from Neisseria meningitidis serogroup B induce mucosal and systemic responses. Methods. 49(4):301-8. doi: 10.1016/j.ymeth.2009.03.025. Bracho, G., Zayas, C., Wang, L., et al. 2009. AFCo1, a meningococcal B-derived cochleate adjuvant, strongly enhances antibody and T-cell immunity against Plasmodium falciparum merozoite surface protein 4 and 5. Malar J. 8:35. doi: 10.1186/1475-2875-8-35. 
Drain, P. K., Nelson, C. M., and Lloyd, J. S. 2003. Single-dose versus multi-dose vaccine vials for immunization programmes in developing countries. Bull World Health Organ. 81(10):726-31.

Faden, H., Modlin, J.F., Thoms, M.L., et al. 1990. Comparative evaluation of immunization with live attenuated and enhanced-potency inactivated trivalent poliovirus vaccines in childhood: systemic and local immune responses. J. Infect. Dis. 162(6):12917.

González, E., Romeu, B., del Campo, J., et al. 2009. Mucosal and systemic immune response against Neisseria meningitidis B induced by single time vaccination strategy. Vaccimonitor. 18 (2):75-77.

Guilherme, L., Postol, E., Freschi de Barros, S. A., et al. 2009. A vaccine against S. pyogenes: design and experimental immune response. Methods. 49(4):316-21. doi: 10.1016/j.ymeth.2009.03.024.

Jennings, G.T. and Bachmann, M.F. 2007. Designing recombinant vaccines with viral properties: a rational approach to more effective vaccines. Curr. Mol. Med. 7(2):143-55. Jiang, W., Gupta, R.K., Deshpande, M.C., et al. 2005. Biodegradable poly(lactic-coglycolic acid) microparticles for injectable delivery of vaccine antigens. Adv Drug Deliv Rev. 57(3):391-410.

Kaul, D. and Ogra, P.L. 1998. Mucosal responses to parenteral and mucosal vaccines. Dev. Biol. Stand. 95:141-6.

Keren, D.F., McDonald, R.A. and Carey, J.L. 1988. Combined parenteral and oral immunization results in an enhanced mucosal immunoglobulin A response to Shigella flexneri. Infect. Immun. 56(4):910-5.. 
Lastre , M., Pérez, O., Labrada, A., et al. 2006. Bacterial derived proteoliposome for allergy vaccines. Vaccine. 24 Supp1 2:S2-34-5.

Lawson, L. B., Norton, E. B. and Clements, J. D. 2011. Defending the mucosa: adjuvant and carrier formulations for mucosal immunity. Current Opinion in Immunology. 23(3):414-20. doi: 10.1016/j.coi.2011.03.009. Lee, C.K, Soike, K., Giannasca, P., et al. 1999. Immunization of rhesus monkeys with a mucosal prime, parenteral boost strategy protects against infection with Helicobacter pylori. Vaccine. 17(23-24):3072-82.

Mann, J.F., Acevedo, R., del Campo, J., et al. 2009. Delivery systems: a vaccine strategy for overcoming mucosal tolerance? Expert Rev Vaccines. 8(1):103-12. doi: $10.1586 / 14760584.8 .1 .103$

Morrison, L.A., Da Costa, X.J. and Knipe, D.M. 1998. Influence of mucosal and parenteral immunization with a replication-defective mutant of HSV-2 on immune responses and protection from genital challenge. Virology. 243(1):178-87.

Neutra, M.R. and Kozlowski, P.A. 2006. Mucosal vaccines: the promise and the challenge. Nat. Rev. Immunol. 6(2):148-58.

O’Hagan, D.T. and Singh, M. 2003. Microparticles as vaccine adjuvant and delivery system. Expert Rev. Vaccines. 2(2):269-83.

Pérez, O., Batista-Duharte, A., González, E., et al. 2012. Human prophylactic vaccine adjuvants and their determinant role in new vaccine formulations. Braz J Med Biol Res. 45(8):681-92..

Pérez, O., Bracho, G., Lastre, M., et al. 2004. Method of obtaining cochlear structures, vaccine compositions, adjuvants and intermediates thereof. WIPO Patent Application WO/2004/047805. 
Pérez, O., González, E., Romeu, B., et al. 2008. Vacunas unitemporales. Patent WO 2010057447 A1.

441

442

443

444

445

446

447

448

449

450

451

452

453

454

455

456

457

458

459

460

Pérez, O., Lastre, M., Lapinet, J., et al. 2001. Immune response induction and new effector mechanisms possibly involved in protection conferred by the Cuban antimeningococcal BC vaccine. Infect Immun. 69(7):4502-8.

Pérez, O., Romeu, B., Cabrera, O., et al. 2013. Adjuvants are Key Factors for the Development of Future Vaccines: Lessons from the Finlay Adjuvant Platform. Front Immunol. 4:407. doi: 10.3389/fimmu.2013.00407..

Pérez, O., Romeu, B., Cabrera, O. et al. 2013 Adjuvants are Key Factors for the Development of Future Vaccines: Lessons from the Finlay Adjuvant Platform. Front Immunol. 4:407. doi: 10.3389/fimmu.2013.00407.

Plotkin, SA. 2005. Vaccines: past, present and future. Nat. Med. 11(4 Suppl):S5-11.

Puga, I., Cols, M., Cerutti, A., et al. 2010. Innate signals in mucosal immunoglobulin class switching. J Allergy Clin Immunol. 126(5):889-95; quiz 896-7. doi: 10.1016/j.jaci.2010.09.026.

Rodríguez, T., Pérez, O., Ménager, N., et al. 2005. Interactions of proteoliposomes from serogroup B Neisseria meningitidis with bone marrow-derived dendritic cells and macrophages: adjuvant effects and antigen delivery. Vaccine. 23(10):1312-21..

Rodríguez, T., Pérez, O., Ugrinovic, S., et al. 2006. Bacterial derived proteoliposome as ideal delivery system and cellular adjuvant. Vaccine. 24 Suppl 2:S2-24-5.

Singh, M., Chakrapani, A. and O’Hagan, D. 2007. Nanoparticles and microparticles as vaccine delivery systems. Expert Rev. Vaccines. 6(5):797-808. 
Treanor, J.J., Mattison, H.R., Dumyati, G., et al. 1992. Protective efficacy of combined

462 live intranasal and inactivated influenza A virus vaccines in the elderly. Ann. Intern.

463 Med. 117(8):625-33.

464 Vajdy, M., Singh, M., Ugozzoli, M., et al. 2003. Enhanced mucosal and systemic 465 immune responses to Helicobacter pylori antigens through mucosal priming followed by 466 systemic boosting immunizations. Immunology. 110(1):86-94.

World Health Organization. 2005. GIVS, Global Immunization Vision and Strategy 2006-2015. Available on the Internet at: www.who.int/vaccinesdocuments/www.unicef.org

World Health Organization. 2009. WHO/UNICEF global estimates for 2008. Available on the Internet at: http://www.who.int/immunization_monitoring/data/en/

Figure 1. PL-specific antibody responses induced by SinTimVaS. Groups of female BALB/c mice $(n=5)$ were SinTimVaS (i.n/i.m) immunized with AFPL1 (i.m route), AFCo1 (i.n route). Control groups included were IN, IM immunized with one, two or three doses with AFPL1 or AFCo1. The PL-specific IgA in their saliva, IgG levels and IgG subtypes in sera were determined using a PL-specific ELISA. (A) Shows the antiPL IgG levels in sera (B) Shows anti-PL IgG subclasses in sera (C) Shows the anti-PL IgA in saliva. Data are expressed as the arbitrary unit + standard deviation of the mean from three independent experiments. Data from subclasses evaluation are expressed as the OD of samples measured at $492 \mathrm{~nm}+$ standard error media (s.e.m) of the means. Significant differences between the means of different groups were determined by a Tukey multiple comparison test using the Graph Pad Prism 4 software (Calif.). A pvalue of $<0.05$ was considered statistically significant. 
Figure 2. PL-specific antibody responses induced by SinTimVaS when other mucosal routes were used. Groups of female BALB/c mice $(n=5)$ were SinTimVaS (i.n/i.m, s.l/i.m or o.r/i.m) immunized with AFPL1 (i.m route), AFCo1 (i.n route). Control groups included were i.n, s.l or o.r immunized with three doses of AFCo1 or two i.m doses of AFPL1. The PL-specific IgA in their saliva, vaginal washes and faeces, IgG levels and IgG subtypes in sera were determined using a PL-specific ELISA. (A) Shows the anti-PL IgG levels in sera (B) Shows anti-PL IgG subclasses in sera (C) Shows the anti-PL IgA in saliva, faeces and vagina. Data are expressed as the arbitrary unit + standard deviation of the mean from three independent experiments. Data from subclasses evaluation are expressed as the OD of samples measured at $492 \mathrm{~nm}+$ s.e.m of the mean. Significant differences between the means of different groups were determined by a Tukey multiple comparison test using the Graph Pad Prism 4 software (Calif.). Each SinTimVaS combination was compared with 3 doses of the related mucosal route or 2 doses of i.m immunization A p-value of $<0.05$ was considered statistically significant and it is represented by different letters, a $(\mathrm{p}<0.001)$; b is $(\mathrm{p}<$

502

Figure 3. PL-specific antibody responses induced by SinTimVaS when subcutaneous priming was used. Groups of female BALB/c mice $(n=5)$ were SinTimVaS (i.n/s.c, s.1/s.c or o.r/s.c) immunized with AFPL1 (s.c route), AFCo1 (i.n route). Control groups included were i.n, s.l or o.r immunized with three doses of AFCo1 or two s.c doses of AFPL1. The PL-specific IgA in their saliva, vaginal washes and faeces, IgG levels and IgG subtypes in sera were determined using a PL-specific ELISA. (A) Shows the anti-PL IgG levels in sera (B) Shows anti-PL IgG subclasses in 
510 sera (C) Shows the anti-PL IgA in saliva, faeces and vagina. Data are expressed as the 511 arbitrary unit + sem of the mean from three independent experiments. Data from 512 subclasses evaluation are expressed as the OD of samples measured at $492 \mathrm{~nm}+$ sem. 513 Significant differences between the means of different groups were determined by a 514 Tukey multiple comparison test using the Graph Pad Prism 4 software (Calif.). Each 515 SinTimVaS combination was compared with 3 doses of the related mucosal route or 2 516 doses of s.c immunization A p-value of $<0.05$ was considered statistically significant and 517 it is represented by different letters, $a(p<0.001)$; $b$ is $(p<0.01)$ and $\mathrm{c}(\mathrm{p}<0.05)$.

Figure 4. Heterologous specific antibody responses induced by SinTimVaS combinations. Groups of female BALB/c mice $(n=5)$ were SinTimVaS (i.n/i.m, s.1/i.m or o.r/i.m) immunized with BSA and TT antigens using AFPL1 (i.m route) and AFCo1 (i.n route) as adjuvants. Control groups included were i.n, s.l or o.r immunized with three doses of AFCo1 plus BSA or TT or two i.m doses of BSA or TT and AFPL1 adsorbed. The BSA or TT specific IgA in their saliva, IgG levels in sera were determined using a BSA or TT specific ELISA. (A) Shows the anti-BSA IgG levels in sera (B) Shows anti-BSA IgA titer in saliva (C) Shows the anti-TT IgG level in sera (D) Shows anti-TT IgA titer in saliva. Data are expressed as OD of samples measured at 492 $\mathrm{nm}+$ s.e.m of the means. Significant differences between the means of different groups were determined by a Tukey multiple comparison test using the Graph Pad Prism 4 software (Calif.). Each SinTimVaS combination was compared with 3 doses of the related mucosal route or 2 doses of i.m immunization A p-value of $<0.05$ was considered 

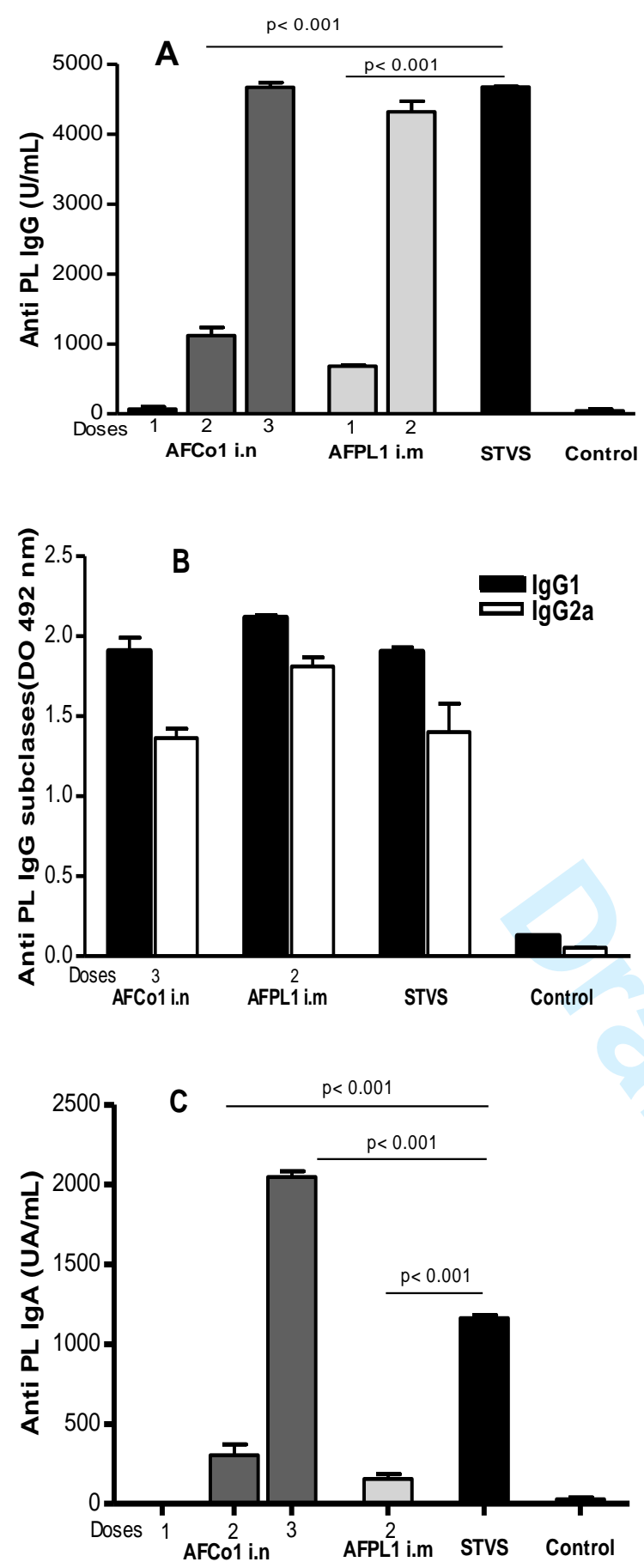

Figure 1. PL-specific antibody responses induced by SinTimVaS. Groups of female BALB/c mice $(n=5)$ were SinTimVaS (i.n/i.m) immunized with AFPL1 (i.m route), AFCo1 (i.n route). Control groups included were i.n, i.m immunized with one, two or three doses with AFPL1 or AFCo1. The PL-specific IgA in their saliva, IgG levels and IgG subtypes in sera were determined using a PL-specific ELISA. (A) Shows the antiPL IgG levels in sera (B) Shows anti-PL IgG subclasses in sera (C) Shows the anti-PL IgA in saliva. Data are expressed as the arbitrary unit + standard deviation of the mean from three independent experiments. Data from subclasses evaluation are expressed as the OD of samples measured at $492 \mathrm{~nm}+$ standard error media (s.e.m) of the means. Significant differences between the means of different groups were determined by a 
Tukey multiple comparison test using the Graph Pad Prism 4 software (Calif.). A pvalue of $<0.05$ was considered statistically significant. 

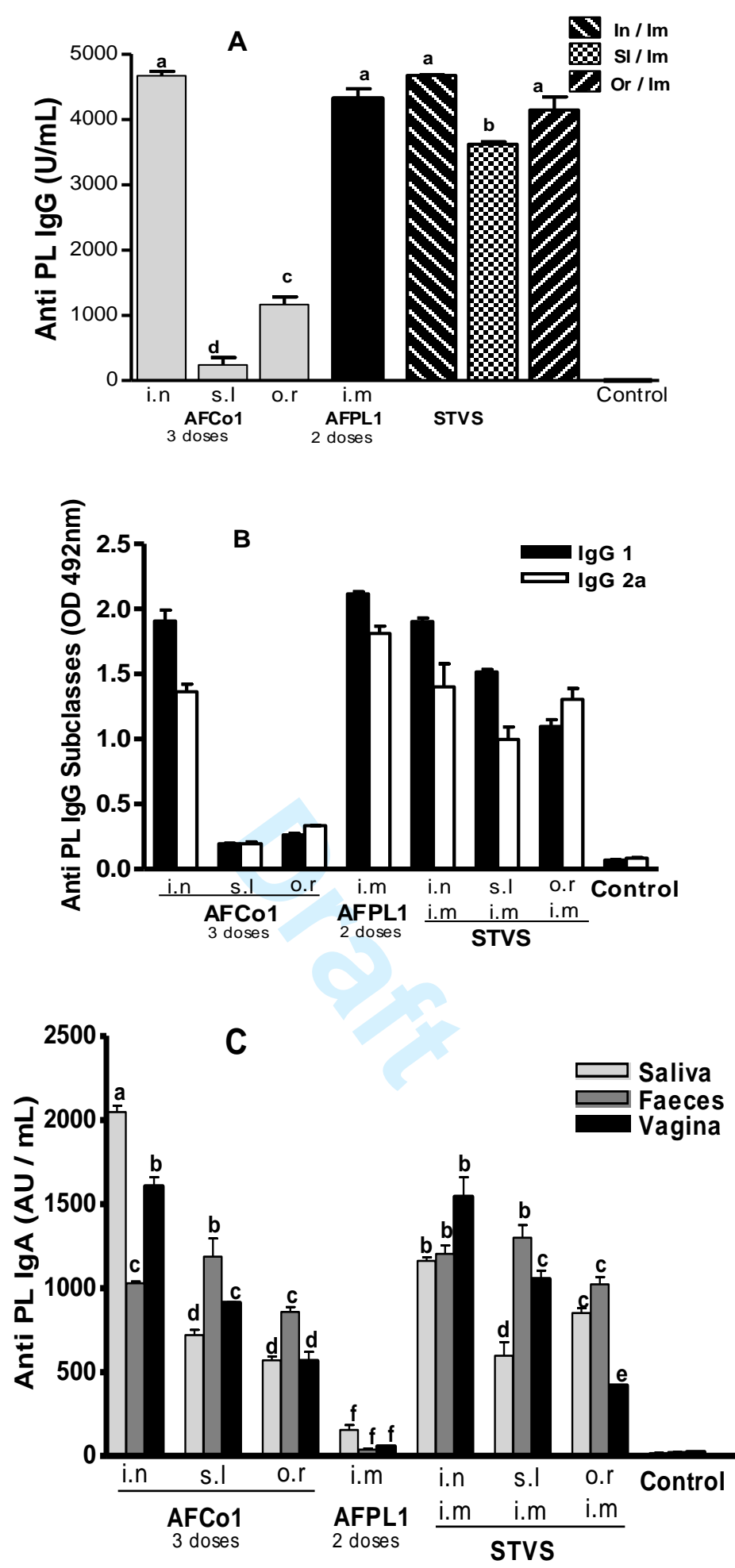

Figure 2. PL-specific antibody responses induced by SinTimVaS when other mucosal routes were used. Groups of female BALB/c mice $(n=5)$ were SinTimVaS (i.n/i.m, s.1/i.m or o.r/i.m) immunized with AFPL1 (i.m route), AFCo1 (i.n route). Control groups included were i.n, s.l or o.r immunized with three doses of AFCo1 or two i.m doses of AFPL1. The PL-specific IgA in their saliva, vaginal washes and faeces, IgG levels and IgG subtypes in sera were determined using a PL-specific ELISA. (A) Shows the anti-PL IgG levels in sera (B) Shows anti-PL IgG subclasses in 
sera (C) Shows the anti-PL IgA in saliva, faeces and vagina. Data are expressed as the arbitrary unit + standard deviation of the mean from three independent experiments. Data from subclasses evaluation are expressed as the OD of samples measured at 492 $\mathrm{nm}+$ s.e.m of the mean. Significant differences between the means of different groups were determined by a Tukey multiple comparison test using the Graph Pad Prism 4 software (Calif.). Each SinTimVaS combination was compared with 3 doses of the related mucosal route or 2 doses of i.m immunization. A p-value of $<0.05$ was considered statistically significant and it is represented by different letters, a ( $\mathrm{p}<$ $0.001)$; $b$ is $(\mathrm{p}<0.01)$ and $\mathrm{c}(\mathrm{p}<0.05)$.
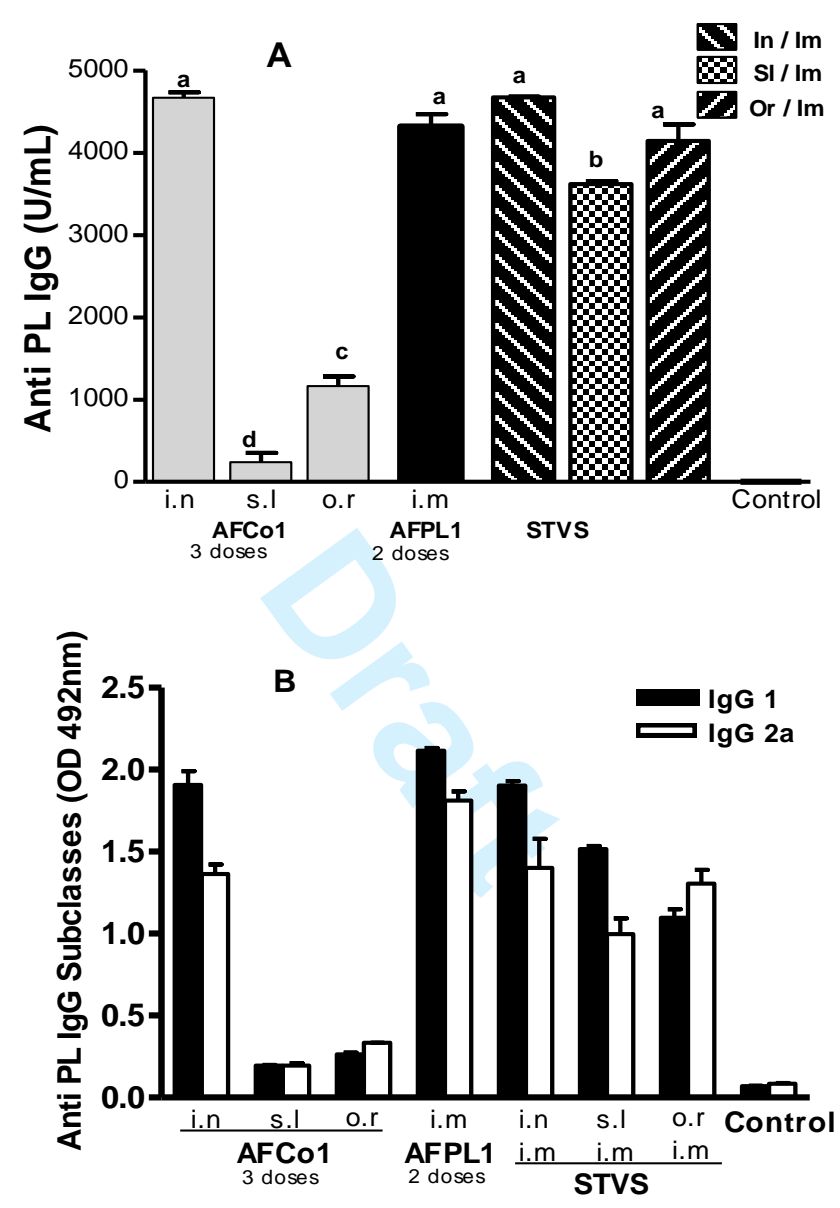


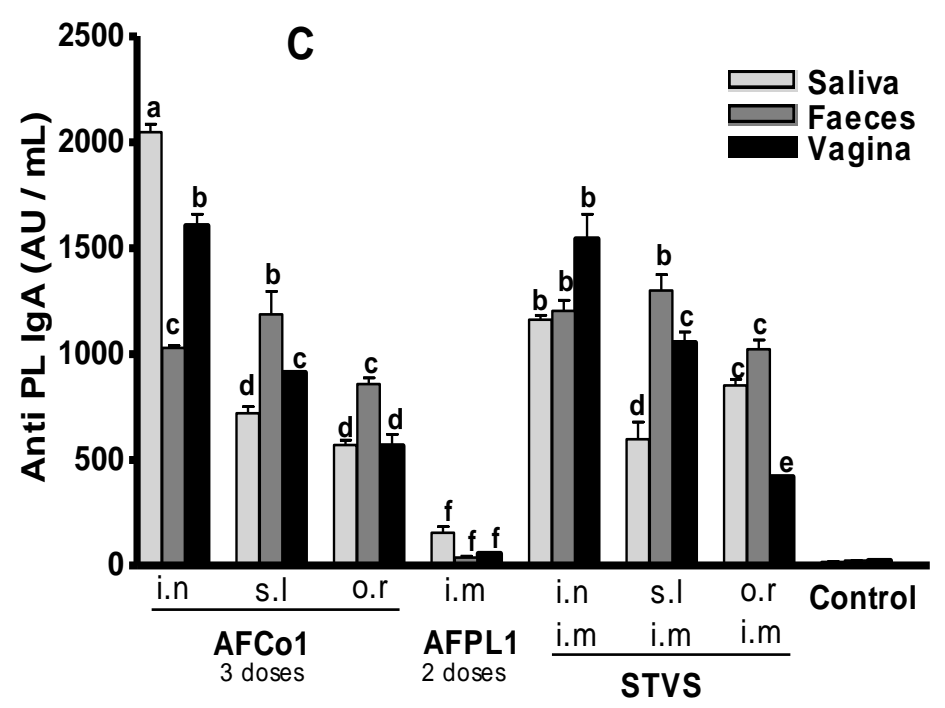

Figure 2. PL-specific antibody responses induced by SinTimVaS when other mucosal routes were used. Groups of female BALB/c mice $(n=5)$ were SinTimVaS (i.n/i.m, s.l/i.m or o.r/i.m) immunized with AFPL1 (i.m route), AFCo1 (i.n route). Control groups included were i.n, s.l or o.r immunized with three doses of AFCo1 or two i.m doses of AFPL1. The PL-specific IgA in their saliva, vaginal washes and faeces, IgG levels and IgG subtypes in sera were determined using a PL-specific ELISA. (A) Shows the anti-PL IgG levels in sera (B) Shows anti-PL IgG subclasses in sera (C) Shows the anti-PL IgA in saliva, faeces and vagina. Data are expressed as the arbitrary unit + standard deviation of the mean from three independent experiments. Data from subclasses evaluation are expressed as the OD of samples measured at 492 $\mathrm{nm}+$ s.e.m of the mean. Significant differences between the means of different groups were determined by a Tukey multiple comparison test using the Graph Pad Prism 4 software (Calif.). Each SinTimVaS combination was compared with 3 doses of the related mucosal route or 2 doses of i.m immunization. A p-value of $<0.05$ was considered statistically significant and it is represented by different letters, a ( $\mathrm{p}<$ $0.001)$; $b$ is $(\mathrm{p}<0.01)$ and $\mathrm{c}(\mathrm{p}<0.05)$. 

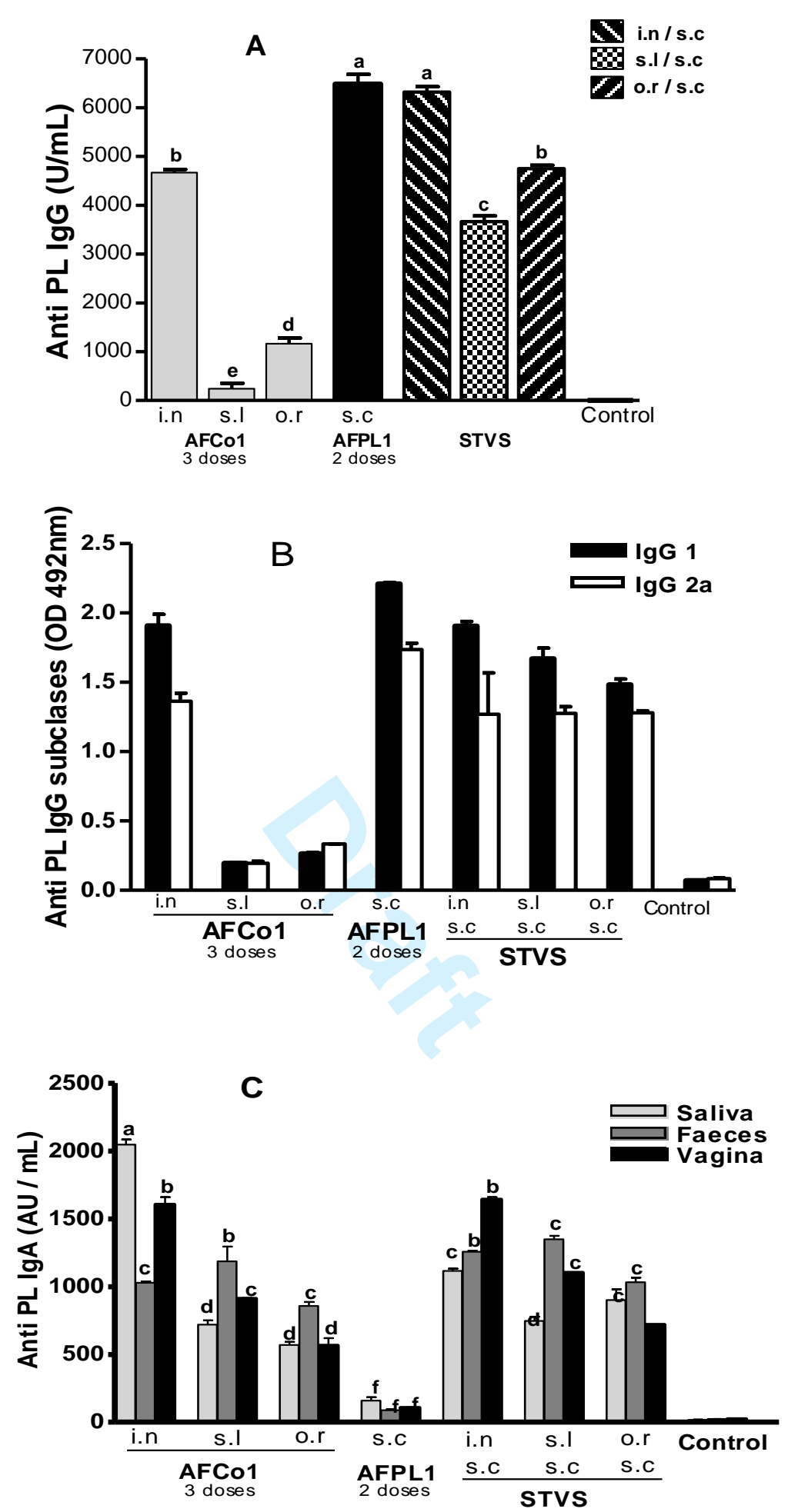

Figure 3. PL-specific antibody responses induced by SinTimVaS when subcutaneous priming was used. Groups of female BALB/c mice $(n=5)$ were SinTimVaS (i.n/s.c, s.1/s.c or o.r/s.c) immunized with AFPL1 (s.c route), AFCo1 (i.n route). Control groups included were i.n, s.l or o.r immunized with three doses of AFCo1 or two s.c doses of AFPL1. The PL-specific IgA in their saliva, vaginal washes and faeces, IgG levels and IgG subtypes in sera were determined using a PL-specific ELISA. (A) Shows the anti-PL IgG levels in sera (B) Shows anti-PL IgG subclasses in sera (C) Shows the anti-PL IgA in saliva, faeces and vagina. Data are expressed as the 
arbitrary unit + sem of the mean from three independent experiments. Data from subclasses evaluation are expressed as the OD of samples measured at $492 \mathrm{~nm}+$ s.e.m. Significant differences between the means of different groups were determined by a Tukey multiple comparison test using the Graph Pad Prism 4 software (Calif.). Each SinTimVaS combination was compared with 3 doses of the related mucosal route or 2 doses of s.c immunization A p-value of $<0.05$ was considered statistically significant and it is represented by different letters, $a(p<0.001)$; $b$ is $(p<0.01)$ and $c(p<0.05)$. 

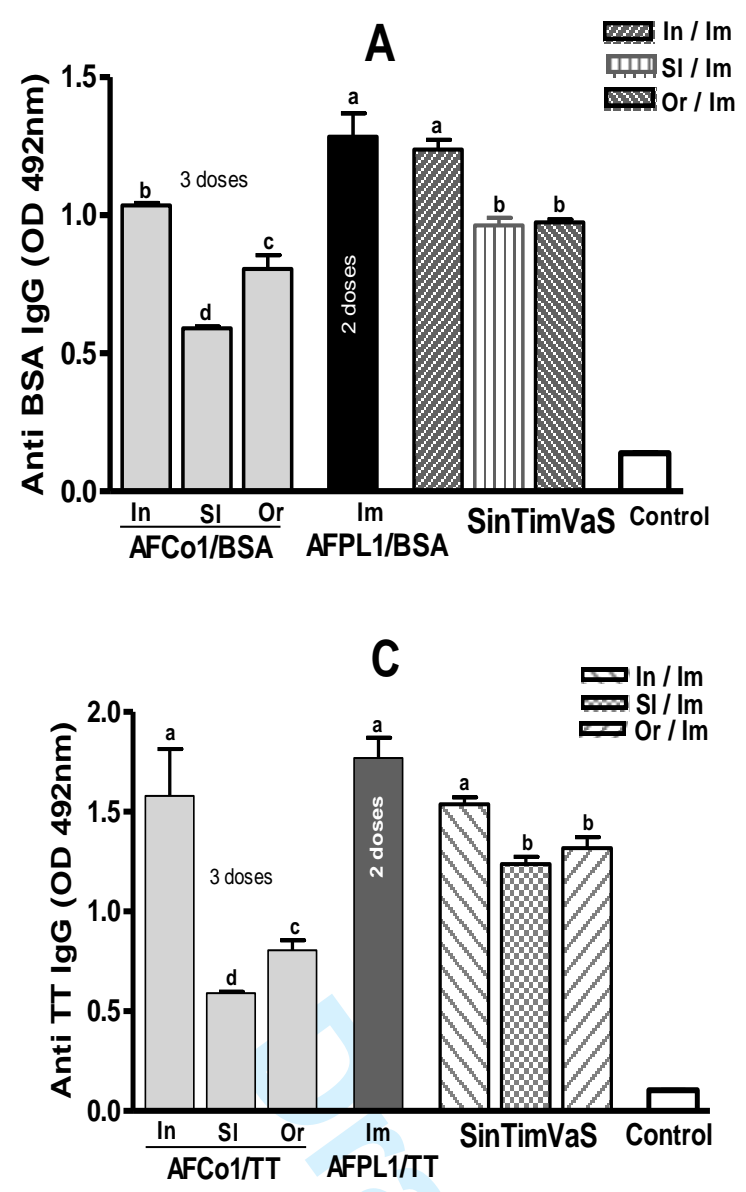

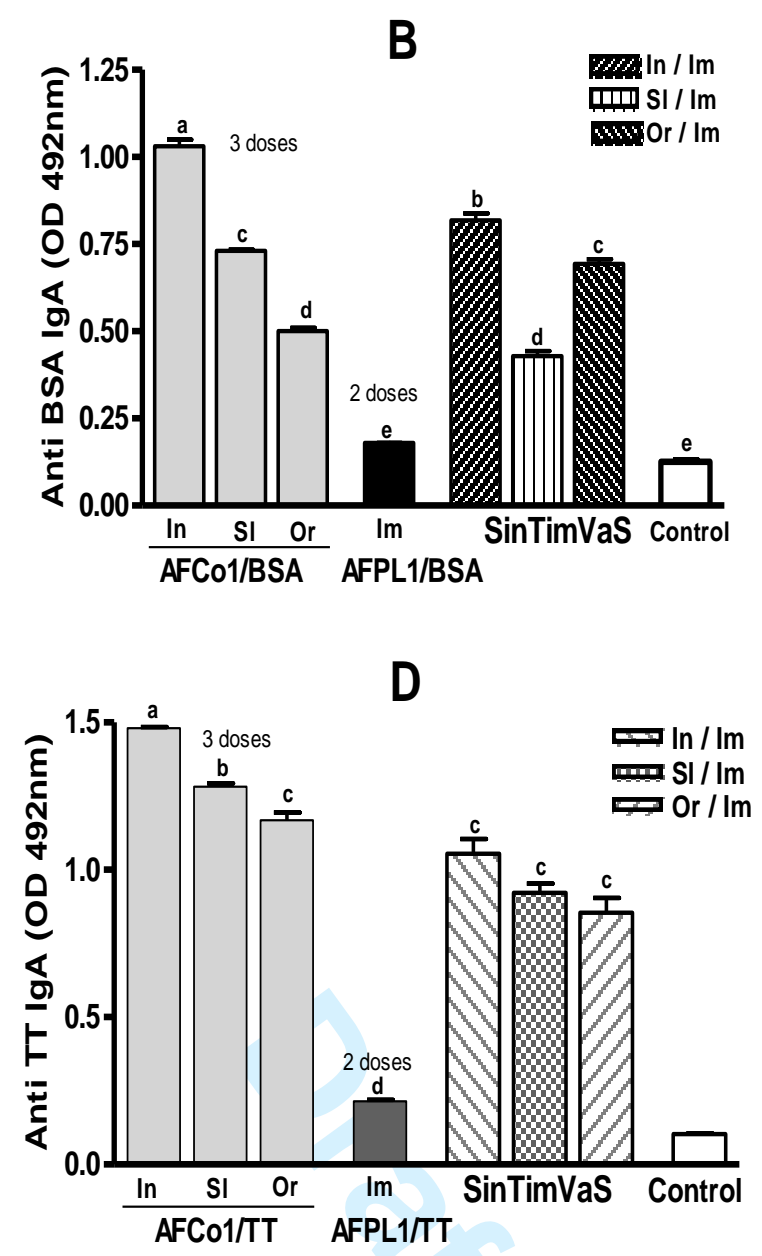

Figure 4. Heterologous specific antibody responses induced by SinTimVaS combinations. Groups of female BALB/c mice $(n=5)$ were SinTimVaS (i.n/i.m, s.l/i.m or o.r/i.m) immunized with BSA and TT antigens using AFPL1 (i.m route) and AFCo1 (i.n route) as adjuvants. Control groups included were i.n, s.l or o.r immunized with three doses of AFCo1 plus BSA or TT or two i.m doses of BSA or TT and AFPL1 adsorbed. The BSA or TT specific IgA in their saliva, IgG levels in sera were determined using a BSA or TT specific ELISA. (A) Shows the anti-BSA IgG levels in sera (B) Shows anti-BSA IgA titer in saliva (C) Shows the anti-TT IgG level in sera (D) Shows anti-TT IgA titer in saliva. Data are expressed as OD of samples measured at $492 \mathrm{~nm}+$ s.e.m of the means. Significant differences between the means of different groups were determined by a Tukey multiple comparison test using the Graph Pad Prism 4 software (Calif.). Each SinTimVaS combination was compared with 3 doses of the related mucosal route or 2 doses of i.m immunization A p-value of $<0.05$ was considered statistically significant and it is represented by different letters, a ( $\mathrm{p}<$ $0.001)$; $b$ is $(p<0.01)$ and $c(p<0.05)$. 


\section{SinTimVaS combinations}

Two priming at the same time, without subsequent boost

$$
\begin{array}{ll}
\text { i.n: AFCo1 + BSA / TT } & \text { i.m: AFPL1 + BSA / TT } \\
\text { s.l: AFCo1 + BSA / TT } & \text { i.m: AFPL1 + BSA / TT } \\
\text { o.r: AFCo1 + BSA /TT } & \text { i.m: AFPL1 + BSA / TT }
\end{array}
$$

* Adjuvants and antigens concentrations used in SinTimVaS were twice those used in traditional parenteral and mucosal routes

Table 1. Single Time Vaccination Combinations used in the studies. 